\title{
ORIGINAL ARTICLE \\ Effects of different bladder management methods on the quality of life in patients with traumatic spinal cord injury
}

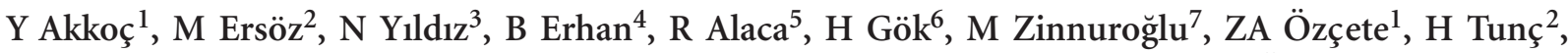 \\ K Kaya ${ }^{3}$, E Alemdaroğlu ${ }^{2}$, M Sarıül ${ }^{2}$, S Konukçu ${ }^{3}$, B Gündüz ${ }^{4}$, AN Bardak ${ }^{4}, S$ Özcan ${ }^{4}$, \\ Y Demir ${ }^{5}$, S Güneş ${ }^{6}$ and $\mathrm{K} \mathrm{Uygunol}^{7}$ for Neurogenic Bladder Turkish Research Group
}

\begin{abstract}
Study design: Multi-center, cross-sectional study.
Objectives: To investigate the effects of different bladder management methods on the quality of life (QoL) in patients with spinal cord injury (SCl).

Setting: Turkey.

Methods: Consecutive SCI patients ( $n=195,74.4 \%$ males), for whom at least 6 months had elapsed since the injury, were included and evaluated in five groups: normal spontaneous micturition (NSM), micturition with assisted maneuvers (MAM), aseptic intermittent catheterization by patient (IC-P), aseptic IC by an attendant/caregiver (IC-A) and indwelling catheterization. The King's Health Questionnaire was used to evaluate the patients' QoL.

Results: The bladder management groups were similar regarding age, time elapsed since injury, education level, marital and occupational status. There was no difference among the groups in general health perception, personal relationships and sleep/energy domain scores. While the NSM group had generally the lowest scores, that is, better QoL, the IC-A group had the highest scores, that is, poorer QoL, in most of the domains. When the patients were grouped according to the frequency of urinary incontinence or American Spinal Injury Association Impairment Scale grades, no difference was found in the domain scores of the groups except the symptom severity domain scores. No significant difference was found between paraplegic and tetraplegic patients in the King's Health Questionnaire domains.
\end{abstract}

Conclusion: The QoL was notably affected in SCI patients in IC-A group and negative effects on emotional status, physical and social activity limitations were observed, as well.

Spinal Cord (2013) 51, 226-231; doi:10.1038/sc.2012.131; published online 13 November 2012

Keywords: spinal cord injury; quality of life; catheterization

\section{INTRODUCTION}

Advances in medical care have increased the survival in patients with spinal cord injury (SCI), and efforts to increase the quality of life (QoL) have gained importance. In the United States, <200000 people live with SCI and $\sim 10000$ new cases occur each year. ${ }^{1}$ Motor vehicle accidents are the most common cause of SCI. Most SCI cases are male, and mean age has been reported to be 37.6 years. ${ }^{2}$ Most patients with SCI have voiding dysfunction; even patients who are able to ambulate may have problems such as urinary incontinence. In addition to the common occurrence of the urinary system complications, voiding dysfunction also leads to social problems by decreasing QoL. Urologic complications have been reported to account for much of the SCI-associated morbidity and as much as $15 \%$ of the SCI-associated mortality. ${ }^{2}$

Patients with SCI generally require lifelong follow-up. Appropriate bladder management method is selected by urologic evaluation during follow-up. The methods utilized include intermittent catheterization (IC), bladder emptying during the Credé's or Valsalva's maneuvers and indwelling catheterization (IDC)..$^{1}$ In the treatment of patients with neurogenic urinary tract dysfunction, medical and physical condition of the patients, as well as their expectations regarding their future social, physical and medical situation should be taken into consideration. Restoration and maintenance of the patient's QoL should remain among the main targets of the treatment. $^{3}$

The present study aimed to investigate the effects of different bladder management methods on the QoL in patients with SCI.

\section{MATERIALS AND METHODS}

Consecutive patients with SCI, for whom at least 6 months had elapsed since the injury and who were capable of comprehending and answering the questions, were included in the present study. The majority of the patients $(70 \%)$ were those who were admitted to outpatient clinics for neuro-urological control visit. The remaining group $(30 \%)$ consisted of the patients who were

${ }^{1}$ Department of Physical Therapy and Rehabilitation, Ege University Faculty of Medicine, İzmir, Turkey; ${ }^{2}$ Department of Physical Medicine and Rehabilitation, Ankara Physical Therapy and Rehabilitation Training and Research Hospital of Ministry of Health, Ankara, Turkey; ${ }^{3}$ Department of Physical Therapy and Rehabilitation, Pamukkale University Faculty of Medicine, Denizli, Turkey; ${ }^{4}$ Department of Physical Medicine and Rehabilitation, Istanbul Physical Therapy and Rehabilitation Training and Research Hospital, İstanbul, Turkey; ${ }^{5}$ Department of Physical Medicine and Rehabilitation, Gülhane Military Medical Academy Turkish Armed Forces Rehabilitation and Care Center, Ankara Turkey; ${ }^{6}$ Department of Physical Therapy and Rehabilitation, Ankara University Faculty of Medicine, Ankara, Turkey and ${ }^{7}$ Department of Physical Therapy and Rehabilitation, Gazi University Faculty of Medicine, Ankara, Turkey

Correspondence: Professor Y Akkoç, Department of Physical Therapy and Rehabilitation, Ege University Faculty of Medicine, İzmir 35100, Turkey.

E-mail: yesim.akkoc@gmail.com

Received 10 April 2012; revised 17 September 2012; accepted 18 September 2012; published online 13 November 2012 
Table 1 General features of the patients grouped according to the bladder management method used

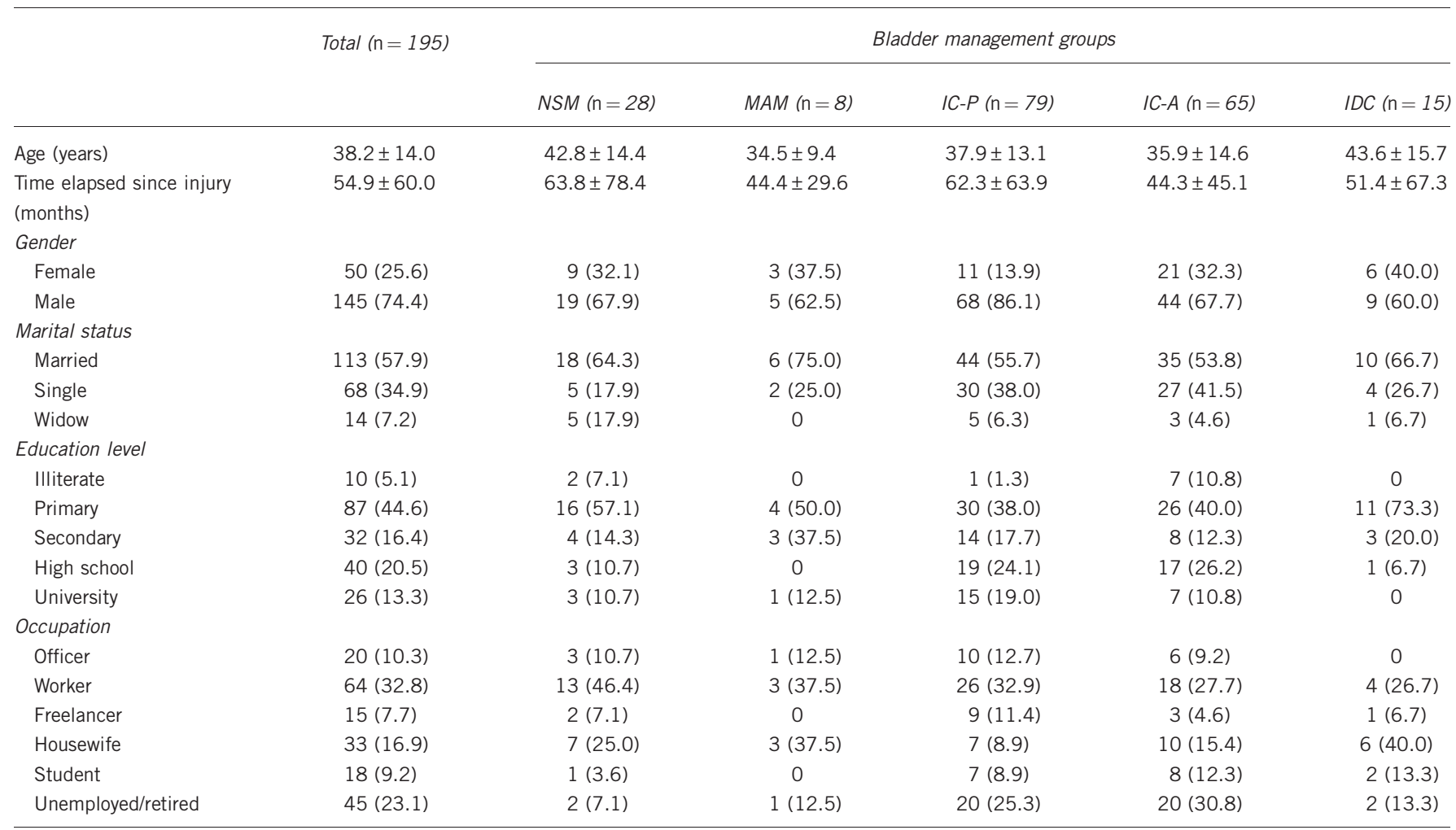

Abbreviations: IC-A, aseptic intermittent catheterization performed by an attendant/caregiver; IC-P, aseptic intermittent catheterization performed by patient; IDC, indwelling catheterization; MAM, micturition with assisted maneuvers; NSM, normal spontaneous micturition.

Data are expressed as mean \pm s.d. or numbers $(\%)$, where appropriate.

hospitalized for a short period of time for neuro-urological re-assessment and further rehabilitation requirements. We certify that all applicable institutional and governmental regulations concerning the ethical use of human volunteers were followed during the course of this research. Patients were evaluated in five groups according to the bladder management method used: (1) normal spontaneous micturition (NSM group), (2) micturition with assisted maneuvers (such as Credé, Valsalva and tapping) (MAM group), (3) aseptic intermittent catheterization performed by patient (IC-P), (4) aseptic intermittent catheterization performed by an attendant/caregiver (IC-A group) and (5) IDC group. Patients with SCI were also classified by the American Spinal Injury Association Impairment Scale (AIS). ${ }^{4}$

The King's Health Questionnaire, the validity of the Turkish version of which was performed by Akkoc et al., ${ }^{5}$ was used to measure the effects of bladder management methods on the patients' QoL. In part 1 of the questionnaire, scores of general health perception (GHP) and incontinence impact (II) on the QoL were calculated. In part 2 of the questionnaire, scores of role limitations (RL), physical limitations (PL), social limitations (SL), personal relationships (PR), emotions (EM), sleep/energy (S/E) and symptom severity (SS) were calculated. Scores range from 0 to 100 ; a score of 100 points indicates the worse health condition (Appendix A).

\section{Statistical analysis}

The PASW (Predictive Analysis Software) Statistics 19.0 (Statistical Package for Social Sciences Inc., Chicago, IL, USA) was used for statistical analysis. Descriptive statistics were expressed as mean, standard deviation, minimum, maximum, and median for numerical variables. Multiple comparisons of independent groups were performed using the Kruskal-Wallis test for nonnormally distributed numerical variables. When the multiple comparisons of independent groups yielded significant results, the Mann-Whitney $U$-test with the Bonferroni correction was used to compare the subgroups. A $P$-value of $<0.05$ was considered as statistically significant.

\section{RESULTS}

In all, 195 patients (145 (74.4\%) males and 50 (25.6\%) females) were included in the present study. The mean age of the study patients was $38.2 \pm 14.0$ years. The mean time elapsed since injury was determined to be $54.9 \pm 60.0$ months. Of the patients, $14.4 \%$ were in the NSM group, $4.1 \%$ were in the MAM group, $40.5 \%$ were in the IC-P group, $33.3 \%$ were in the IC-A group and $7.7 \%$ were in the IDC group. General features of the patients in the groups are presented in Table 1.

There was no difference among the groups in terms of mean age $(P=0.091)$, time elapsed since injury $(P=0.170)$, marital status $(P=0.223)$, education level $(P=0.055)$ or occupational status $(P=0.228)$. A statistically significant difference was found among the groups regarding gender distribution $(P=0.040)$. The proportion of male patients was higher in all groups, with the highest proportion $(86.1 \%)$ in the IC-P group.

The most common cause of injury was traffic accidents with a rate of $39.3 \%$, which was followed by fall from height $(36.1 \%)$ and jumping into the sea (7.9\%). Injury levels and the AIS grades of the patients in the bladder management groups are presented in Table 2.

The mean scores of the study groups in the King's Health Questionnaire are presented in Table 3. Higher scores in the King's Health Questionnaire represent a worse QoL.

No difference was found among the bladder management groups with respect to their scores in GHP, PR and S/E domains. The paired group comparisons performed using the Mann-Whitney $U$-test revealed that the mean II scores of the IC-P and IC-A groups were significantly higher than that of NSM group $(P=0.004$ and $P=0.001$, respectively). The mean RL score of the IC-A group was significantly higher than that of the NSM group $(P=0.003)$. The 
mean PL score of the IC-A group was significantly higher compared with the scores of both the NSM and IC-P groups $(P<0.001$ and $P=0.005$, respectively). The mean scores of the MAM, IC-A and IDC groups in SL domain were significantly higher than that of the NSM group ( $P=0.008, P=0.004$ and $P=0.014$, respectively). The mean EM score of the IC-A group was significantly higher than that of the NSM group $(P=0.001)$. The mean scores of the IC-P and IC-A groups in SS domain were significantly higher than that of the NSM group ( $P=0.001$ and $P<0.001$, respectively). The mean SS score of the IC-A group was found to be significantly higher than that of the IDC group $(P=0.034)$.

While the NSM group had generally the lowest scores, that is, better QoL, the IC-A group had the highest scores, that is, poorer QoL, in most of the domains.

Table 2 Neurological injury level and the American Spinal Injury Association Impairment Scale (AIS) grades of the patients in the bladder management groups

\begin{tabular}{|c|c|c|c|c|c|c|}
\hline & \multirow{2}{*}{$\begin{array}{c}\text { Total } \\
(\mathrm{n}=195)\end{array}$} & \multicolumn{5}{|c|}{ Bladder management groups } \\
\hline & & $\begin{array}{c}N S M \\
(\mathrm{n}=28)\end{array}$ & $\begin{array}{l}M A M \\
(\mathrm{n}=8)\end{array}$ & $\begin{array}{c}I C-P \\
(\mathrm{n}=79)\end{array}$ & $\begin{array}{c}I C-A \\
(\mathrm{n}=65)\end{array}$ & $\begin{array}{c}I D C \\
(\mathrm{n}=15)\end{array}$ \\
\hline \multicolumn{7}{|c|}{ Neurological level of the injury } \\
\hline Cervical & $42(21.5)$ & $7(25.0)$ & 0 & $1(1.3)$ & $29(44.6)$ & $5(33.3)$ \\
\hline Thoracic & $110(56.4)$ & $11(39.3)$ & $7(87.5)$ & $61(77.2)$ & $26(40.0)$ & $5(33.3)$ \\
\hline Lumbar & $43(22.1)$ & $10(35.7)$ & $1(12.5)$ & $17(21.5)$ & $10(15.4)$ & $5(33.3)$ \\
\hline \multicolumn{7}{|l|}{ AIS grades } \\
\hline A & $98(50.3)$ & $2(7.1)$ & $3(37.5)$ & $45(57.0)$ & $40(61.5)$ & $8(53.3)$ \\
\hline B & $30(15.4)$ & $2(7.1)$ & $0(0.0)$ & $14(17.7)$ & 11 (16.9) & $3(20.0)$ \\
\hline C & $29(14.9)$ & $6(21.4)$ & $0(0.0)$ & $11(13.9)$ & $9(13.8)$ & $3(20.0)$ \\
\hline $\mathrm{D}$ & 35 (17.9) & $16(57.1)$ & $5(62.5)$ & $8(10.1)$ & $5(7.7)$ & $1(6.7)$ \\
\hline$E$ & $3(1.5)$ & $2(7.1)$ & $0(0.0)$ & $1(1.3)$ & $0(0.0)$ & $0(0.0)$ \\
\hline
\end{tabular}

The patients were regrouped to evaluate the effect of urinary incontinence status on the QoL. Of the patients, $44.1 \%(n=86)$ did not have urinary incontinence, $34.9 \%(n=68)$ had urinary incontinence several times a day, $13.8 \%(n=27)$ had urinary incontinence several times a week and $7.2 \%(n=14)$ had urinary incontinence several times a month. The mean scores of the patients regrouped according to urinary incontinence status in the King's Health Questionnaire are presented in Table 4.

There was a significant difference among urinary incontinence groups only in SS scores. The paired group comparisons performed using the Mann-Whitney $U$-test revealed that the mean SS scores of patients with urinary incontinence several times a day and those with urinary incontinence several times a week were found to be significantly higher compared with that of patients without urinary incontinence $(P<0.001$ and $P=0.018$, respectively).

Effects of the AIS grades of the patients on the QoL are presented in Table 5. There was no significant difference among the AIS groups in the King's Health Questionnaire domains, except in SS scores. Moreover, tetraplegic $(n=44)$ and paraplegic $(n=151)$ patients were compared in terms of the King's Health Questionnaire domains in the present study using the Mann-Whitney $U$-test, no significant difference was found between these two group of patients in all domains of the King's Health Questionnaire $(P>0.05$ for each).

\section{DISCUSSION}

In addition to providing proper medical care to patients with SCI, the QoL also needs to be improved for these patients to cope with and adapt to their changing life style after the injury. ${ }^{6}$ Increased life expectancy with the improvements in health care and the need for a lifelong follow-up in patients with SCI necessitates a more comprehensive approach to maintain physical, psychological and social well-being of these patients. Psychosocial assessment together with regular medical examinations is required to determine the factors reducing the QoL and to develop corrective actions toward these factors.

Voiding dysfunction, which is observed in most patients with SCI, is associated with an increase in complications and a decrease in the

Table 3 Effects of bladder management methods on the quality of life

\begin{tabular}{|c|c|c|c|c|c|c|}
\hline & \multicolumn{5}{|c|}{ Bladder management groups } & \multirow[t]{2}{*}{$P^{a}$} \\
\hline & $N S M(\mathrm{n}=28)$ & MAM $(\mathrm{n}=8)$ & $I C-P(\mathrm{n}=79)$ & $I C-A(\mathrm{n}=65)$ & $I D C(\mathrm{n}=15)$ & \\
\hline GHP & $33.5 \pm 23.0$ & $41.0 \pm 29.1$ & $33.3 \pm 21.5$ & $41.6 \pm 28.2$ & $40.3 \pm 30.6$ & 0.415 \\
\hline II & $37.1 \pm 36.3^{b, c}$ & $63.0 \pm 31.7$ & $55.9 \pm 35.5^{d}$ & $61.3 \pm 34.9^{d}$ & $56.0 \pm 31.7$ & 0.014 \\
\hline $\mathrm{RL}$ & $31.3 \pm 34.3^{c}$ & $52.4 \pm 32.9$ & $41.3 \pm 35.2$ & $53.4 \pm 36.6^{d}$ & $48.6 \pm 32.5$ & 0.024 \\
\hline$P L$ & $29.5 \pm 34.3^{c}$ & $56.6 \pm 40.0$ & $40.0 \pm 33.8^{c}$ & $57.5 \pm 35.7^{b, d}$ & $50.5 \pm 30.9$ & 0.001 \\
\hline SL & $29.2 \pm 32.0^{c, e, f}$ & $68.9 \pm 37.2^{d}$ & $43.8 \pm 35.7$ & $49.6 \pm 34.6^{d}$ & $53.2 \pm 34.0^{d}$ & 0.009 \\
\hline PR & $57.1 \pm 40.6$ & $48.4 \pm 41.2$ & $45.4 \pm 35.8$ & $52.1 \pm 34.9$ & $58.3 \pm 40.5$ & 0.883 \\
\hline EM & $22.2 \pm 30.3^{c}$ & $44.8 \pm 29.4$ & $33.6 \pm 27.3$ & $45.9 \pm 33.7^{d}$ & $40.3 \pm 33.5$ & 0.006 \\
\hline$S / E$ & $14.1 \pm 22.0$ & $18.9 \pm 22.5$ & $23.7 \pm 25.1$ & $26.6 \pm 25.7$ & $22.6 \pm 21.0$ & 0.061 \\
\hline SS & $22.2 \pm 22.2^{b, c}$ & $49.5 \pm 32.1$ & $41.6 \pm 27.3^{d}$ & $48.8 \pm 28.1^{\mathrm{d}, \mathrm{f}}$ & $32.2 \pm 25.0^{c}$ & 0.001 \\
\hline
\end{tabular}

Abbreviations: IC-A, aseptic intermittent catheterization performed by an attendant/caregiver; IC-P, aseptic intermittent catheterization performed by patient; IDC, indwelling catheterization; EM, emotions; GHP, general health perception; II, incontinence impact; MAM, micturition with assisted maneuvers; NSM, normal spontaneous micturition; PL, physical limitations; PR, personal relationships; RL, role limitations; S/E, sleep/energy; SL, social limitations; SS, symptom severity.

Data are expressed as mean \pm s.d.

$P$-values less than 0.05 are indicated in bold.

aKruskal-Wallis test.

bDifferent from the IC-P group.

cDifferent from the IC-A group.

Different from the IC-A group.

NSM group.

eDifferent from the MAM group.

fDifferent from the IDC group. 
QoL. In a longitudinal, observational, epidemiologic study performed on a neurogenic bladder population $(n=46271)$ encompassing 4168 persons with SCI, due to the observation of high incidences of urinary tract complications and hospitalizations among patients with neurogenic bladder, it was concluded that these patients might have suboptimal management. ${ }^{7}$ In their study, Hicken et al. ${ }^{8}$ reported that individuals with impaired bowel and bladder control had lower QoL on several domains than individuals with independent control of bowel and bladder. In the light of this information, it is obvious that bladder management is one of the important components of the treatment in patients with SCI. In the consortium guideline, various recommendations have been established for bladder management for adults with SCI; ${ }^{1}$ however, to our knowledge, studies on the QoL are lacking. In the present study, we discussed impaired bladder function interfering with the QoL in patients with

\section{Table 4 Effect of frequency of urinary incontinence on the quality of} life

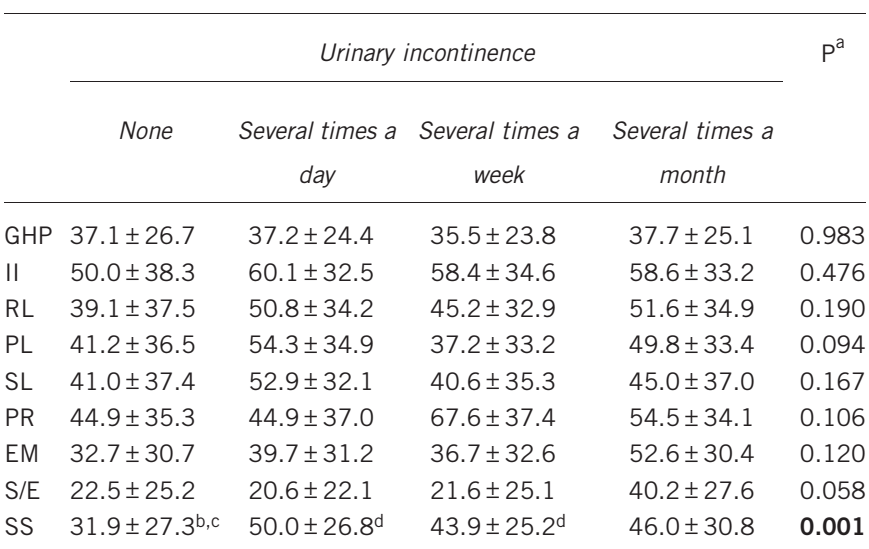

Abbreviations: EM, emotions; GHP, general health perception; II, incontinence impact; $\mathrm{PL}$, physical limitations; PR, personal relationships; RL, role limitations; S/E, sleep/energy; SL, social limitations; SS, symptom severity.

Data are expressed as mean \pm s.d.

$P$-values less than 0.05 are indicated in bold.

akruskal-Wallis test.

bDifferent from 'several times a day' group.

'Different from 'several times a week' group.

'Different from 'none' group.
SCI, and we investigated the relationship between bladder management method and the QoL.

In determining the proper bladder management method, several factors have a role such as physical capacity and sociocultural status of the patient, the level and severity of injury, the living environment of the patient, and whether the patient is receiving care or not; the ideal method for an individual patient may vary in time. In a study evaluating the selection of bladder management in the United States between 1972 and 2005, it was reported that among bladder management methods, clean intermittent catheterization (CIC) increased in popularity with time; however, of the patients initially on CIC, only $20 \%$ remained on this form of bladder management. ${ }^{9}$ In their study in which bladder management methods were compared, Cameron et al. ${ }^{10}$ reported patients on IDC to be associated with more medical complications and lower levels of participation as compared with patients using other bladder management methods (spontaneous voiding, condom catheterization and intermittent catheterization).

For individualization of the treatment, different treatment options have been attempted, and reports have been published about their impact on the QoL. ${ }^{11-16}$

Pannek and Kullik ${ }^{13}$ evaluated 41 patients with lower urinary tract dysfunction caused by SCI using the Qualiveen questionnaire and urodynamic tests and reported that the Qualiveen scale ratings regarding fears and feelings were significantly worse in patients with suboptimal bladder function than in patients in whom treatment success was achieved. They suggested that a treatment regimen leading to favorable urodynamic outcome and continence was associated with better QoL. In their study, Oh et al. ${ }^{14}$ evaluated 132 patients and 150 controls using the Medical Outcomes Study Short Form-36 General Health Survey (SF-36) to investigate the QoL in patients on CIC due to neurogenic bladder secondary to SCI; they concluded that patients on CIC generally exhibited a reduced QoL in all health domains. It has been reported that comparing patients on CIC with patients using other bladder management methods (such as IDC, suprapubic catheter, suprapubic tapping, straining or external collecting devices) in addition to comparing with the general population, as well as using specific questionnaires rather than general health questionnaires might yield more significant results. ${ }^{17}$ In this regard, we evaluated the QoL of patients with

Table 5 Effects of the American Spinal Injury Association Impairment Scale (AIS) grades of the patients on the quality of life

\begin{tabular}{|c|c|c|c|c|c|c|}
\hline & \multicolumn{5}{|c|}{ AIS groups } & \multirow[t]{2}{*}{$\mathrm{P}^{\mathrm{a}}$} \\
\hline & $A(\mathrm{n}=98)$ & $B(\mathrm{n}=30)$ & $C(\mathrm{n}=29)$ & $D(\mathrm{n}=35)$ & $E(\mathrm{n}=3)$ & \\
\hline GHP & $37.8 \pm 24.8$ & $31.4 \pm 28.5$ & $37.6 \pm 25.6$ & $39.1 \pm 24.5$ & $33.3 \pm 14.4$ & 0.587 \\
\hline II & $61.1 \pm 33.0$ & $53.1 \pm 37.5$ & $44.2 \pm 37.2$ & $49.8 \pm 37.6$ & $61.1 \pm 42.0$ & 0.090 \\
\hline$R L$ & $48.6 \pm 35.6$ & $45.3 \pm 36.8$ & $35.9 \pm 30.2$ & $42.5 \pm 40.2$ & $38.8 \pm 25.5$ & 0.475 \\
\hline$P L$ & $50.4 \pm 34.2$ & $39.4 \pm 39.5$ & $40.1 \pm 32.1$ & $42.6 \pm 38.6$ & $53.7 \pm 44.6$ & 0.377 \\
\hline SL & $48.8 \pm 34.7$ & $39.3 \pm 36.3$ & $40.6 \pm 32.9$ & $45.5 \pm 39.0$ & $40.7 \pm 44.9$ & 0.712 \\
\hline PR & $50.2 \pm 36.2$ & $38.7 \pm 33.5$ & $61.3 \pm 35.1$ & $45.6 \pm 39.9$ & $61.1 \pm 55.0$ & 0.573 \\
\hline EM & $37.9 \pm 30.1$ & $38.5 \pm 30.9$ & $34.3 \pm 33.3$ & $34.8 \pm 34.6$ & $51.8 \pm 32.0$ & 0.718 \\
\hline$S / E$ & $21.0 \pm 21.8$ & $24.4 \pm 21.9$ & $25.9 \pm 27.5$ & $23.3 \pm 30.7$ & $41.6 \pm 38.2$ & 0.427 \\
\hline SS & $48.8 \pm 26.4^{b, c}$ & $40.9 \pm 31.3$ & $30.4 \pm 24.7^{d}$ & $28.5 \pm 26.7^{d}$ & $36.3 \pm 28.9$ & $<0.001$ \\
\hline
\end{tabular}

Abbreviations; EM, emotions; GHP, general health perception; II, incontinence impact; PL, physical limitations; PR, personal relationships; RL, role limitations; S/E, sleep/energy; SL, social limitations; SS, symptom severity.

Data are expressed as mean $\pm \mathrm{s} . \mathrm{d}$.

$P$-values less than 0.05 are indicated in bold.

akruskal-Wallis test.

bDifferent from the group $C$

'Different from the group $D$.

dDifferent from the group A. 
NSM, those urinating with assisted maneuvers, patients on IC performed by the patient him/herself or by the caregiver/attendant, and those using IC. For this purpose, we used the King's Health Questionnaire in the present study.

In their study on 142 patients with SCI, Liu et al. ${ }^{15}$ evaluated the relationship between bladder management methods and healthrelated QoL using the SF-36 and the King's Health Questionnaire. They reported normal voiding in only $21 \%$ of their patients with SCI, whereas $79 \%$ were reported to be on other forms of bladder management. The researchers suggested that the type of bladder management might affect the health-related QoL in patients with SCI and determined that CIC performed by an attendant, indwelling transurethral catheterization and indwelling suprapubic catheterization were the three groups associated with the worst mental status. They also confirmed that the frequency of incontinence had a strong impact on HRQL. In the present study, the rate of patients with NSM was $14.4 \%$, and the remaining $85.6 \%$ were using various types of bladder management. Similarly to the results reported in the study by Liu et al., ${ }^{15}$ we determined that the bladder management groups had poorer QoL as compared with the patients with normal micturition and that the highest scores (poorer QoL) were observed in patients on IC performed by an attendant/caregiver. When the effect of the frequency of incontinence on QoL was evaluated, patients with urinary incontinence several times a day and those with urinary incontinence several times a week were found to have higher scores in SS domain compared with patients without urinary incontinence. No significant difference was noted in the other QoL domains among the groups formed according to frequency of urinary continence. When the confounding factors that could affect the QoL no significant difference was noted among the bladder management groups with respect to age, time elapsed since injury, marital status, education level and occupational status. The rate of male patients was significantly higher in the IC-P group, and significant differences were observed among the groups with respect to the injury levels and AIS grades. However, there were no significant differences in the domain scores of the King's Health Questionnaire, except the SS domain score, among the AIS grade groups. Additionally, no significant difference was found between the paraplegic and tetraplegic patients in the King's Health Questionnaire domains. These findings suggested us that the bladder management groups were comparable in terms of the QoL.

In a study conducted in Spain by Sanchez Raya et al., ${ }^{16}$ the QoL of 91 patients with SCI was evaluated by comparing different bladder management techniques. They evaluated the patients in three different groups according to the bladder management method regularly used by the patients: intermittent catheterization, condom catheter or indwelling catheter. The higher scores (poorer QoL) in the King's Health Questionnaire were reported for patients using indwelling catheters. The authors also stated that patients treated with condom catheters had the best QoL according to the King's Health Questionnaire scores; however, no significant differences were reported as compared with the other urinary management techniques.

In the present study, patients in the NSM group had the best QoL scores (lowest scores in the questionnaire), whereas patients in the ICA group had the highest scores (poorer QoL). Scores of the IC-A group in II, RL, PL, SL, EM and SS domains were significantly higher than that of the NSM group. In other words, bladder dysfunction negatively affected the QoL in patients on IC performed by an attendant/caregiver; in addition to negative effects on emotional status in this patient group, RL and limited physical and social activity were also observed.

\section{Study limitations}

The number of patients was not homogeneously distributed among groups in our study, which can be considered as a limitation. Moreover, urinary complications were not addressed in the present study.

\section{CONCLUSIONS}

In conclusion, it was determined in patients with SCI that those with normal micturition had better QoL compared with those using bladder management methods and patients experiencing the highest distress and limitations were those using IC performed by an attendant/caregiver. Bladder management is of importance to improve the QoL in patients with SCI. To judge the most appropriate bladder management method, further large-scale studies are required.

\section{DATA ARCHIVING}

There were no data to deposit.

\section{CONFLICT OF INTEREST}

The authors declare no conflict of interest.

1 Consortium for Spinal Cord Medicine. Bladder management for adults with spinal cord injury: a clinical practice guideline for health-care providers. J Spinal Cord Med 2006; 29: 527-573.

2 Fonte N. Urological care of the spinal cord-injured patient. J Wound Ostomy Continence Nurs 2008; 35: 323-333.

3 Stöhrer M, Blok B, Castro-Diaz D, Chartier-Kastler E, Del Popolo G, Kramer G et al. EAU guidelines on neurogenic lower urinary tract dysfunction. Eur Urol 2009; 56 81-88.

4 American Spinal Injury Association. International Standards for Neurological Classification of Spinal Cord Injury. reprint 2008. American Spinal Injury Association: Atlanta, GA, 2008.

5 Akkoc Y, Karapolat H, Eyigor S, Yesil H, Yüceyar N. Quality of life in multiple sclerosis patients with urinary disorders: reliability and validity of the Turkish version of King's Health Questionnaire. Neurol Sci 2011; 32: 417-421.

$6 \mathrm{Ku} \mathrm{JH}$. The management of neurogenic bladder and quality of life in spinal cord injury. BJU Int 2006; 98: 739-745.

7 Manack A, Motsko SP, Haag-Molkenteller C, Dmochowski RR, Goehring Jr EL, NguyenKhoa BA et al. Epidemiology and healthcare utilization of neurogenic bladder patients in a US claims database. Neurourol Urodyn 2011; 30: 395-401.

8 Hicken BL, Putzke JD, Richards JS. Bladder management and quality of life after spinal cord injury. Am J Phys Med Rehabil 2001; 80: 916-922.

9 Cameron AP, Wallner LP, Tate DG, Sarma AV, Rodriguez GM, Clemens JQ. Bladder management after spinal cord injury in the United States 1972 to 2005. J Urol 2010; 184: 213-217

10 Cameron AP, Wallner LP, Forchheimer MB, Clemens JQ, Dunn RL, Rodriguez G et al. Medical and psychosocial complications associated with method of bladder management after traumatic spinal cord injury. Arch Phys Med Rehabil 2011; 92: 449-456.

11 Sugimura T, Arnold E, English S, Moore J. Chronic suprapubic catheterization in the management of patients with spinal cord injuries: analysis of upper and lower urinary tract complications. BJU Int 2008; 101: 1396-1400.

12 Vastenholt JM, Snoek GJ, Buschman HP, van der Aa HE, Alleman ER, Ijzerman MJA 7-year follow-up of sacral anterior root stimulation for bladder control in patients with a spinal cord injury: quality of life and users' experiences. Spinal Cord 2003; 41: 397-402.

13 Pannek J, Kullik B. Does optimizing bladder management equal optimizing quality of life? Correlation between health-related quality of life and urodynamic parameters in patients with spinal cord lesions. Urology 2009; 74: 263-266.

14 Oh SJ, Ku JH, Jeon HG, Shin HI, Paik NJ, Yoo T. Health-related quality of life of patients using clean intermittent catheterization for neurogenic bladder secondary to spinal cord injury. Urology 2005; 65: 306-310.

15 Liu CW, Attar KH, Gall A, Shah J, Craggs M. The relationship between bladder management and health-related quality of life in patients with spinal cord injury in the UK. Spinal Cord 2010; 48: 319-324.

16 Sánchez RJ, Romero CG, González VMA, Ramírez GL, García FL, Conejero Sugrañes J Quality of life evaluation in spinal cord injured patients comparing different bladder management techniques. Actas Urol Esp 2010; 34: 537-542.

17 Akkoc Y. Health-related quality of life of patients using clean intermittent catheterization for neurogenic bladder secondary to spinal cord injury. Urology 2005; 66: 1360 . 
APPENDIX: A

The King's Health Questionnaire (Part 1: Questionnes 1-2 and Part

\section{2: Questionnes 3-8)}

1. How would you describe your health at the present?

Please tick one answer

Very good

Good

Fair

Poor

Very poor

2. How much do you think your bladder problem affects your life?

Please tick one answer

Not at all

A little

Moderately

A lot

Below are some daily activities that can be affected by bladder problems.

How much does your bladder problem affect you?

We would like you to answer every question. Simply tick the number that applies to you

\begin{tabular}{lllll} 
& 1 & 2 & 3 & 4 \\
\hline 3. ROLE LIMITATIONS & $\begin{array}{l}\text { Not } \\
\text { at all }\end{array}$ & & lightly & Moderately \\
A
\end{tabular}

A. Does your bladder problem affect your house-

hold tasks?

(cleaning, shopping, and so on)

B. Does your bladder problem affect your job, or

your normal daily activities outside the home?

\begin{tabular}{lllll}
1 & 2 & 3 & 4 \\
\hline 4. PHYSICALSOCIAL LIMITATION & Not & Slightly & Moderately & A
\end{tabular}

4. PHYSICAL/SOCIAL LIMITATION

Not Slightly Moderately A

A Does your bladder problem affect your physical at all

lot

activities (e.g., going for a walk, running, sport,

gym etc)?

B. Does your bladder problem affect your ability

to travel?

C. Does your bladder problem limit your social

life?

D. Does your bladder problem limit your ability to

see and visit friends?

\begin{tabular}{llllll} 
& 0 & 1 & 2 & 3 & 4 \\
\hline 5. PERSONAL RELATIONSHIPS & $\begin{array}{l}\text { Not } \\
\text { Applicable }\end{array}$ & Not all & Slightly & Moderately & A \\
& lot
\end{tabular}

A. Does your bladder problem affect your relationship with your partner?

B. Does your bladder problem affect your sex life?

C. Does your bladder problem affect your family life?

\begin{tabular}{lllll}
1 & 2 & 3 & 4 \\
\hline 6. EMOTIONS & $\begin{array}{l}\text { Not at Slightly } \\
\text { all Moderately }\end{array}$ & $\begin{array}{l}\text { Very } \\
\text { much }\end{array}$
\end{tabular}

A. Does your bladder problem make you feel depressed?

B. Does your bladder problem make you feel depressed?

C. Does your bladder problem make you feel bad about yourself?

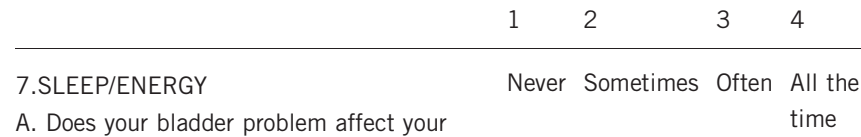
sleep?

B. Does your bladder problem make you feel worn out and tired?

8.Do you do any of the following? If so how

much?

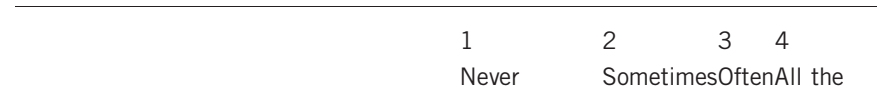
A. Wear pads to keep dry?
B. Be careful how much fluid you drink?
C. Change your underclothes because they
get wet?
D. Worry in case you smell? 